EXTENDED REPORT

\title{
Contrast and glare testing in keratoconus and after penetrating keratoplasty
}

\author{
K Pesudovs, P Schoneveld, R J Seto, D J Coster
}

Br J Ophthalmol 2004;88:653-657. doi: 10.1136/bjo.2003.027029

See end of article for authors' affiliations

.....................

Correspondence to: K Pesudovs, Department of Ophthalmology, Flinders Medical Centre, Bedford Park, South Australia, 5042, Australia; Konrad. Pesudovs@ flinders.edu.au

Accepted for publication 22 August 2003

\begin{abstract}
Aim: To compare the performance of keratoconus, penetrating keratoplasty (PK), and control subjects on clinical tests of contrast and glare vision, to determine whether differences in vision were independent of visual acuity (VA), and thereby establish which vision tests are the most useful for outcome studies of PK for keratoconus.

Methods: All PK subjects had keratoconus before graffing and no subjects had any other eye disease. The keratoconus ( $n=11$, age 35.0 (SD 11.1) years), forme fruste keratoconus $(n=6,33.0$ (13.0)), PK $(n=21$, $41.2(7.9))$, and control $(n=24,33.7(8.6))$ groups were similar in age. Vision testing, conducted with optimal refractive correction in place, included low contrast visual acuity (LCVA) and Pelli-Robson contrast sensitivity (PRCS) both with and without glare, as well as VA.

Results: Normal subjects saw better than PK subjects who in turn saw better than keratoconus subjects on all raw measures. However, when adjusted for VA, the normal group only saw significantly better than the keratoconus group on LCVA (low contrast loss $0.05(0.04) \vee 0.15(0.12), F_{2,48}=6.16 ; p<0.01$, post hoc Sheffé $p<0.05$ ), and the decrements to glare were no worse than for normals. The forme fruste keratoconus group were indistinguishable from normals on all measures.

Conclusions: PK subjects have superior vision to keratoconus subjects, but not as good as normal subjects. Including mild keratoconus subjects within a keratoconus group could confound these differences in vision. While VA is an excellent test for comparing normal, keratoconus and PK groups, additional information can be provided by LCVA and PRCS, but not by glare testing. Outcomes research into keratoconus management should include a measure in the contrast domain.
\end{abstract}

$\mathrm{K}$ eratoconus was first described by Nottingham in 1854, and has been comprehensively reviewed by several authors since. ${ }^{2-6}$ Visual acuity (VA) has been the mainstay of assessing the progression of the severity of keratoconus and the effectiveness of treatment..$^{7-9}$ Visual acuity is also an important factor in determining the change in treatment method from spectacles to rigid contact lenses and on to penetrating keratoplasty (PK). ${ }^{3}$ However, the inadequacy of high contrast VA as an indicator of visual quality is widely appreciated. ${ }^{10}$

Several studies have investigated other measures of vision in keratoconus. Most of these have looked at contrast sensitivity (CS), ${ }^{11-22}$ and some have demonstrated that the loss of contrast sensitivity in keratoconus cannot be predicted from VA measurement. ${ }^{12}{ }^{13} 18$ The value of low contrast visual acuity (LCVA) testing in keratoconus is less clear. While LCVA has shown to be reduced in keratoconus, ${ }^{914}$ 19 $^{23-25}$ the independence of this reduction from VA has not been established.

Penetrating keratoplasty has been demonstrated to be an effective treatment for keratoconus, as measured by an improvement in VA. ${ }^{7}$ However, contrast vision after PK for keratoconus has not been so well studied. Contrast sensitivity after PK is usually better than in keratoconus, ${ }^{15-17} 19222627$ but not in all studies. ${ }^{27-29}$ Further research on this seems warranted. Low contrast visual acuity in PK has had minimal investigation with one study reporting some abnormal values, ${ }^{19}$ and another showing no difference from keratoconus eyes. ${ }^{28}$ Glare testing in keratoconus and PK subjects has been the subject of several small studies that have shown glare losses in keratoconus greater than in normals or PK subjects. ${ }^{16} 172230$ However, two studies have shown glare losses greater after PK than in keratoconus. ${ }^{15}{ }^{16}$ This confusion is at odds with the clinical impression that patients benefit from PK. This inconsistency seems worthy of further investigation.

In this study, LCVA and CS, with and without glare, were measured and compared across age similar groups of keratoconus, PK, forme fruste keratoconus, and normal subjects. This was done to determine whether LCVA detected losses in keratoconus subjects that were independent of VA, to add to the published data on contrast vision in PK subjects, and to investigate the value of glare testing in keratoconus and PK subjects. Vision was assessed using standard clinical tests so that those which proved most useful could then be used for routine clinical testing, or incorporated into a protocol for PK for keratoconus outcomes research. The study groups were selected to be uncomplicated, stable, and typical of the condition and, in addition, a forme fruste keratoconus group was included to investigate the potential confounding effect of disease severity sampling on comparing keratoconus subjects with PK and normal subjects.

\section{SUBJECTS AND METHODS}

Informed consent was obtained from all subjects after the nature of the study had been fully explained. The tenets of the Declaration of Helsinki were followed and the study gained approval from the Flinders University ethics committee. Inclusion criteria were age 15 years or older, and normal healthy eyes with a VA better than 0.1 logMAR (6/7.5 Snellen equivalent) for the control group, or keratoconus (for the keratoconus group), or having undergone PK for keratoconus with an uncomplicated postoperative course (for example, no

Abbreviations: CS, contrast sensitivity; GLLVCA, low contrast visual acuity glare loss; GLPRCS, Pelli-Robson contrast sensitivity glare loss; LCL, low contrast loss; LCVA, low contrast visual acuity; PK, penetrating keratoplasty; PRCS, Pelli-Robson contrast sensitivity; VA, visual acuity 
rejection, cataract development, etc) of at least 12 months (for the PK group). Keratoconus was defined by the staging system of Krumeich et al as being at least stage 1 (eccentric corneal steepening, induced myopia/astigmatism $<5 \mathrm{D}$, corneal radii $<48 \mathrm{D}$, Vogt's striae, no scars). ${ }^{31}$ Subjects with probable mild keratoconus as suggested by a history of keratoconus or having had PK for keratoconus in the other eye, as well as abnormal retinoscopy but not meeting the criteria for stage 1 , were included as a separate forme fruste keratoconus group.

Exclusion criteria were any ocular pathology (other than keratoconus for the keratoconus group) or abnormality such as amblyopia and strabismus, any previous ocular surgery (other than penetrating keratoplasty for the PK group), any neurological problem, systemic disease, or taking of any medication which may affect contrast sensitivity, inability to speak English sufficiently to be instructed to perform the tests, insufficient mental ability to perform the tests, and physical disability which would make it arduous to perform the tests (for example, wheelchair bound). Subjects with keratoconus and PK for keratoconus were drawn from the anterior segment clinic of the Department of Ophthalmology at Flinders Medical Centre (FMC) on a consecutive attendance basis. Control subjects were drawn from medical students and staff of FMC.

Data were collected on the visual performance of 11 eyes of 11 keratoconus subjects, six eyes of six forme fruste keratoconus subjects, 21 eyes of 21 PK subjects, and 24 eyes of 24 normal subjects. The subjects had a mean age of 36.4 (SD 9.8) years. Data were collected in a single session. All subjects were refracted and optimally corrected before data collection. For the subjects who normally wore contact lenses (keratoconus 8/11, PK 7/21, normal 2/24) refraction and optimal correction took place over their contact lenses. The measurements taken were $\log$ MAR high contrast visual acuity, LCVA, Pelli-Robson contrast sensitivity (PRCS), LCVA with glare, and PRCS with glare.

Both VA and LCVA were measured on a computerised monitor based system. ${ }^{32}$ The program utilises the psychophysical "staircase" method, using a forced choice protocol, to determine the acuity end point, which is taken as the average of 13 staircase reversals. This offers excellent reliability and validity and is free from learning effects. The program uses the same $5 \times 4$ letters used in Bailey-Lovie logMAR charts and the results are given in $\log M A R .{ }^{32}$ Low contrast visual acuity is analogous to VA testing except that the target optotypes are reduced in contrast. Twenty five per cent (Weber) contrast optotypes were utilised since this has been reported to be the most suitable contrast level for detecting visual loss in early cataracts under glare conditions and thus may be suitable for keratoconus subjects. ${ }^{33}$ Testing was conducted at 3.0 metres and the monitor had a maximum luminance of $185 \mathrm{~cd} / \mathrm{m}^{2}$. Low contrast visual acuity was also measured under glare conditions (LCVAglare).

There is no accepted standardised method for glare testing. In this study, LCVA and Pelli-Robson contrast sensitivity were measured with and without the presence of a light source directed at the subject. The glare source consisted of two projection lamps placed either side of the test chart and monitor. The baseline room illuminance was 80 lux. The luminance of the projector sources was $1800 \mathrm{~cd} / \mathrm{m}^{2}$. Natural pupils were used and care taken to ensure neither occlusion of the glare source nor macular photostress occurred. ${ }^{34}$

The Pelli-Robson contrast sensitivity (PRCS) chart was used according to previously published methodology. ${ }^{35}{ }^{36}$ This test was chosen from among other available clinical charts for its superior validity and reliability ${ }^{36}{ }^{37}$ especially compared to sinusoidal grating charts such as the Vistech and FACT. ${ }^{38}$ The use of a letter reading task for both CS and LCVA testing also allowed easy comparison of the two techniques. The PelliRobson chart was positioned 3 metres from the subject and had a luminance of $100 \mathrm{~cd} / \mathrm{m}^{2}$. The test was scored by the modified method of letter by letter scoring, ${ }^{39}$ and results were given in units of $\log$ contrast sensitivity $(\log C S)$. Contrast sensitivity was also measured under the same glare conditions (PRCSglare) as LCVAglare.

The results are reported as raw measures and three derived measures of contrast and glare loss. Taking the simple arithmetic difference between glare and no glare, or high and low contrast demonstrates the effect of contrast or glare independent of baseline VA or CS. This facilitates comparison of groups of disease versus no disease where matching for VA is not possible. Low contrast loss (LCL) is defined as the difference between the high contrast VA and LCVA: LCL $=$ LCVA - VA. $^{40}{ }^{41}$ Low contrast visual acuity glare loss $\left(\mathrm{GL}_{\mathrm{LVCA}}\right)$ is defined as the difference between the LCVAglare and LCVA: GL LCVA $_{\text {LCVAglare }}-$ LCVA. $^{40}$ Pelli-Robson contrast sensitivity glare loss (GL $\mathrm{L}_{\mathrm{PRCS}}$ ) is defined as the difference between PRCS and PRCSglare: $\mathrm{GL}_{\mathrm{PRCS}}=\mathrm{PRCS}-$ PRCSglare. ${ }^{40}$

One way analyses of variance (ANOVA) with Sheffé post hoc significance testing were used to compare groups. All statistical analyses were performed on the SPSS software package v10.0 (SPSS Inc, Chicago, IL, USA).

\section{RESULTS}

The results of visual performance of the normal control group and the study groups are listed in table 1. The three groups were similar for age $\left(\mathrm{F}_{2,53}=3.32 ; \mathrm{p}>0.05\right)$. The normal controls performed better on all raw vision measures than both the keratoconus and PK groups. Similarly, the PK group performed better than the keratoconus group. However, as the groups were not matched for VA $\left(\mathrm{F}_{2,48}=30.60 ; \mathrm{p}<0.001\right)$, they were also compared using three difference measures. Only two significantly different results were identified. The keratoconus group had a greater low contrast loss than the normal group $\left(0.15(0.12) v 0.05(0.04), \mathrm{F}_{2,48}=6.16 ; \mathrm{p}<0.01\right.$, post hoc Sheffé $\mathrm{p}<0.05$ ) - that is, the measurement of LCVA identified loss of vision in the keratoconus group not predicted by VA testing. Conversely, the keratoconus group had a smaller glare loss with the Pelli-Robson contrast sensitivity chart than the normal group $(-0.16(0.36) \vee 0.07$ (0.11), $\mathrm{F}_{2,49}=4.23 ; \mathrm{p}<0.05$, post hoc Sheffé $\left.\mathrm{p}<0.05\right)$ - that is, the vision in the normal group worsened under glare but improved in the keratoconus group. On all three difference measures the PK group was indistinguishable from the normal and keratoconus groups. Low contrast visual acuity and CS testing did identify visual loss in keratoconus and PK not appreciated with VA testing, but glare testing did not provide any such useful information.

To examine the influence of disease severity on vision loss in the keratoconus group, a forme fruste keratoconus group was also tested. This group was age similar (33.0 (13.0) years; $\mathrm{p}>0.05)$ and was also indistinguishable from normals on all measures of vision (VA -0.15 (0.04) p $>0.05$; LCVA -0.06 (0.08) $\mathrm{p}>0.05$; LCVAglare -0.03 (0.09) $\mathrm{p}>0.05$; PRCS 1.65 (0.12) $\mathrm{p}>0.05$; PRCSglare 1.65 (0.15) $\mathrm{p}>0.05$; LCL 0.10 $(0.05) \mathrm{p}>0.05 ; \mathrm{GL}_{\mathrm{LCVA}} 0.03(0.09) \mathrm{p}>0.05 ; \mathrm{GL}_{\mathrm{PRCS}} 0.00(0.13)$ $\mathrm{p}>0.05)$.

\section{DISCUSSION}

The normal control group performed significantly better on all raw measures of vision compared to the keratoconus and PK groups. However, this chiefly reflects that the normal and study groups were not matched for VA. When adjusting for VA differences by using the difference measures low contrast loss and glare loss, the normal group was only significantly better on LCL (logMAR drop between VA and LCVA) and 
Table 1 Mean (SD) for the five raw vision measures* and three difference measurest in normals, keratoconus, and penetrating keratoplasty subjects

\begin{tabular}{|c|c|c|c|c|}
\hline & Normal $(n=24)$ & Keratoconus $(n=11)$ & PK $(n=21)$ & Statistics \\
\hline Age & $33.7(8.6)$ & $35.0(11.1)$ & $41.2(7.9)$ & \\
\hline Visual acuity & $-0.18(0.05)$ & $0.17(0.22)$ & $-0.03(0.11)$ & $\begin{array}{l}\left(\mathrm{F}_{2,48}=30.60 ; \mathrm{p}<0.001 \text { post hoc }\right. \\
\text { normal }<\text { keratoconus, } \mathrm{p}<0.001 \text {, } \\
\text { normal }<\mathrm{PK}, \mathrm{PK}<\text { keratoconus, } \mathrm{p}<0.01)\end{array}$ \\
\hline $\begin{array}{l}\text { Low contrast visual } \\
\text { acuity }\end{array}$ & $-0.12(0.06)$ & $0.29(0.23)$ & $0.10(0.12)$ & $\begin{array}{l}\left(F_{2,48}=41.70 ; p<0.001 \text { post hoc }\right. \\
\text { normal }<\text { keratoconus, normal }<P K \\
p<0.001, P K<\text { keratoconus, } p<0.01)\end{array}$ \\
\hline $\begin{array}{l}\text { Low contrast visual } \\
\text { acuity under glare }\end{array}$ & $-0.11(0.08)$ & $0.35(0.28)$ & $0.08(0.14)$ & $\begin{array}{l}\left(\mathrm{F}_{2,48}=31.87 ; \mathrm{p}<0.001 \text { post hoc }\right. \\
\text { normal }<\text { keratoconus, normal }<\mathrm{PK} \text {, } \\
\mathrm{p}<0.001, \mathrm{PK}<\text { keratoconus, } \mathrm{p}<0.01)\end{array}$ \\
\hline Pelli-Robson contrast sensitivity & $1.80(0.13)$ & $1.10(0.28)$ & $1.39(0.22)$ & $\begin{array}{l}\left(F_{2,51}=50.69 ; p<0.001 \text { post hoc }\right. \\
\text { normal }>\text { keratoconus, normal }>P K \text {, } \\
p<0.001 \text {, } P K>\text { keratoconus, } p<0.01)\end{array}$ \\
\hline Pelli-Robson contrast sensitivity under glare & $1.73(0.11)$ & $1.25(0.46)$ & $1.41(0.21)$ & $\begin{array}{l}\left(F_{2,49}=17.09 ; p<0.001 \text { post hoc }\right. \\
\text { normal }>\text { keratoconus, } p<0.001 \text {, } \\
\text { normal }>P K, p<0.01)\end{array}$ \\
\hline Low contrast loss & $0.05(0.04)$ & $0.15(0.12)$ & $0.12(0.09)$ & $\begin{array}{l}\left(F_{2,48}=6.16 ; p<0.01 \text { post hoc }\right. \\
\text { normal }<\text { keratoconus, } p<0.05)\end{array}$ \\
\hline Glare loss (low contrast visual acuity) & $0.02(0.08)$ & $0.06(0.11)$ & $-0.02(0.08)$ & $\left(F_{2,49}=4.23 ; p<0.05\right.$ post hoc \\
\hline Glare loss (Pelli-Robson contrast sensitivity) & $0.07(0.11)$ & $-0.16(0.36)$ & $-0.01(0.21)$ & keratoconus $>$ normal, $p<0.05$ ) \\
\hline
\end{tabular}

*Visual acuity (VA), low contrast visual acuity (LCVA), low contrast visual acuity under glare (LCVAglare) (all in logMAR), Pelli-Robson contrast sensitivity (PRCS), Pelli-Robson contrast sensitivity measured under glare (PRCSglare) (both in logCS).

thow contrast loss = LCVA - VA, low contrast glare loss = LCVAglare - LCVA, PRCS glare loss = PRCS - PRCSglare.

only compared to the keratoconus group (normal $0.05(0.04)$, keratoconus $0.15(0.12) ; \mathrm{p}<0.05)$. The PK group was indistinguishable from both normal and keratoconus groups on these three difference measures. This suggests that VA is a very effective measure for distinguishing between normal, keratoconus, and PK subjects, but that CS and LCVA do provide some additional information.

Previous studies on keratoconus subjects have found reduced LCVA compared to normal subjects, but none of these studies controlled for VA by looking at LCL. ${ }^{14} 19$ 23-25 However, deriving LCL from published VA and LCVA data suggests there is slightly greater LCL in keratoconus subjects compared to normals (at 10\% but not 30\% contrast), ${ }^{14}$ slightly more LCL in keratoconus subjects with apical corneal scarring compared to keratoconus subjects without scarring, ${ }^{23}{ }^{24}$ and greater LCL in more severe keratoconus. ${ }^{25}$ While these reports support our findings, these differences were not tested statistically. Similarly, previous studies where CS was measured in keratoconus found reduced CS compared to normals, in some cases these losses were shown to be independent of VA. ${ }^{11-22}$ Two of these studies measured PRCS in keratoconus. ${ }^{21}{ }^{22}$ Rose et al found smaller losses of PRCS than we did, which may have been because they reported binocular measurements, or because their keratoconus group were less impaired than ours, which is possible since they gave no definition of keratoconus, and all subjects were able to habitually wear their optimal correction. ${ }^{21}$ Conversely, Brahma et al's group had much worse PRCS and VA than ours, but this was because their group were all presenting for surgery and were therefore unable to achieve satisfactory visual correction. ${ }^{22}$ This conclusion is supported by their VA and PRCS data for the other eye, which were better than our keratoconus data, although these data were a mix of 12 keratoconus and six PK eyes. ${ }^{22}$ Another possible reason for differences between studies is a difference in the relative proportions of those wearing RGP contact lenses, as these have been shown to improve contrast sensitivity in keratoconus compared to spectacle wearers. ${ }^{13} 42$

Low contrast visual acuity data in PK subjects has previously been shown to include subnormal values in one series, ${ }^{19}$ and to be indistinguishable from keratoconus eyes in another. ${ }^{28}$ Subnormal CS after PK for keratoconus has also been shown previously. ${ }^{15-17} 1926$ Two longitudinal studies of keratoconus subjects before and after PK both found improved CS after PK. ${ }^{22}$ Several cross sectional studies comparing PK to keratoconus eyes support this finding. ${ }^{17} 172629$ Our data consolidate these findings, in that subjects with fully healed PK have CS better than keratoconus subjects, but not as good as normals, and LCVA losses which do not exceed normals. Those studies where PK subjects have been shown to have greater deficits in CS than keratoconus subjects were either in the early postoperative period, ${ }^{27}{ }^{28}$ or during a rejection episode. ${ }^{29}$

The two glare loss measures did not yield any further vision losses in the keratoconus and PK groups, not identified with LCVA or PRCS testing alone. For GL $\mathrm{L}_{\mathrm{LCVA}}$, all groups were similar, but for $\mathrm{GL}_{\mathrm{PRCS}}$, the normal group $(0.07$ (0.11)) was significantly worse than the keratoconus group $(-0.16$ (0.36); $\mathrm{F}_{2,49}=4.23 ; \mathrm{p}<0.05$, post hoc Sheffé $\left.\mathrm{p}<0.05\right)$. While the keratoconus group did better than the normal group in terms of the change in CS under glare conditions the raw PRCS under glare scores were still worse in the keratoconus group compared to the normals $(1.41(0.21) v 1.73(0.11)$; $\mathrm{F}_{2,49}=17.09 ; \mathrm{p}<0.001$, post hoc Sheffé $\left.\mathrm{p}<0.001\right)$. That glare testing is of no value in either keratoconus or PK reflects that the main mechanism of visual loss in keratoconus and PK is optical aberration rather than light scatter. ${ }^{19}$ Our same experimental set-up finds large glare losses in cataract since the main mechanism of optical disturbance from cataract is forward light scatter. ${ }^{40}$ If the main problem is aberrations, visual performance must be pupil dependent. ${ }^{44}$ Therefore, the use of natural pupils, which will constrict in the presence of a bright glare source, reduces the chance of finding decreased visual performance under glare and, in this study, probably explains the significant increase in PRCS in the presence of glare for the keratoconus group. This raises the possibility that glare testing with a less bright glare source may be more useful in keratoconus. Alternatively, instead of testing vision under glare, perhaps low illumination testing, such as with the Smith Kettlewell Institute Low Luminance (SKILL) Card, ${ }^{45}$ which could allow use of maximum natural pupil size and hence sample more aberrated optics, may be more appropriate in keratoconus.

The only longitudinal study of keratoconus subjects before and after PK $(\mathrm{n}=18)$, in which glare testing was done (using the Brightness Acuity Tester (BAT) with VA and PRCS 
charts), found glare losses for keratoconus but not for PK. ${ }^{22}$ The reason for the difference to our results may be that the BAT glare source is not as bright as our projector glare source, thus may cause less pupillary constriction. Two small cross sectional studies ( $\mathrm{n}=6$ and 7 ) also showed a trend toward CS deficit under glare for keratoconus subjects compared with normal subjects. ${ }^{16}{ }^{17}$ Conversely, two small studies ( $\mathrm{n}=4$ and 5 ) found glare losses in PK greater than those seen in keratoconus. ${ }^{15} 16$ These small studies may have included keratoconus cases with apical corneal scarring, which has been shown to cause forward light scatter and measurable glare loss. ${ }^{24}{ }^{30}$ Similarly, the PK series may have included cases with graft oedema, perhaps early in the postoperative recovery, which could also cause glare loss. Therefore, while glare testing is not useful for measuring vision in all cases of keratoconus or PK, it may still be useful in the presence of apical corneal scarring, or graft oedemafor example, in the first month after surgery ${ }^{27}$ or after a rejection episode. ${ }^{29}$

Defining the point along a spectrum from normal to disease state at which the transition from one entity to the other occurs is not a difficulty confined to keratoconus. ${ }^{46}$ However, for keratoconus this difficulty has led to debate over the existence of unilateral keratoconus, ${ }^{47}$ issues for study design, ${ }^{48}$ and difficulty determining patient suitability for laser refractive surgery. ${ }^{49}$ For this study we defined keratoconus according to an accepted, but conservative system. ${ }^{31}$ To illustrate that the inclusion of more mild cases of keratoconus could confound the results, we also tested a group of six forme fruste keratoconus subjects. This group were indistinguishable from the normal group on all vision measures. If we had included these subjects in the keratoconus group then the results of this study would have been different. This highlights the problem of sampling disease severity for cross sectional design studies. This problem could be avoided by using a longitudinal outcome study design. Indeed, the clinical impression that PK patients see better than keratoconus patients arises from comparing pre-PK keratoconus patients who want visual improvement to their post-PK state. Such pre-PK keratoconus subjects often proceed to surgery because they are unable to tolerate optimum refractive correction, so it is possible that this clinical impression is exaggerated by undercorrection of refractive error. Thus it is important in longitudinal study execution to ensure vision testing is conducted with optimal refraction in place.

While PK provides vision superior to keratoconus, it does not achieve levels of vision as good as normals. The pursuit of improvement in the surgical management of keratoconus requires vision testing to establish these improvements. Based on our findings, studies of treatments for keratoconus should include a measure of contrast vision, either PRCS or LCVA.

\section{ACKNOWLEDGEMENTS}

KP is supported by NHMRC Sir Neil Hamilton Fairley Fellowship 0061.

\section{Authors' affiliations}

K Pesudovs, P Schoneveld, R J Seto, D J Coster, Department of Ophthalmology, Flinders Medical Centre and Flinders University, Bedford Park, South Australia, 5042, Australia

\section{REFERENCES}

1 Nottingham J. Practical observations on conical cornea: and on the short sight, and other defects of vision connected with it. London: John Churchill: Liverpool: Deighton \& Laughton, 1854:1-19.

2 Krachmer JH, Feder RS, Belin MW. Keratoconus and related noninflammatory corneal thinning disorders. Surv Ophthalmol 1984;28:293-322.
3 Lawless M, Coster DJ, Phillips AJ, et al. Keratoconus: diagnosis and management. Aust NZ J Ophthalmol 1989;17:33-60.

4 Feder RS. Noninflammatory ectatic disorders. In: Krachmer JH, Mannis MJ, Holland EJ, eds. Cornea: cornea and external disease: clinical diagnosis and management. St Louis: Mosby, 1997;2:1091-106.

5 Maguire LJ. Ectatic corneal degenerations. In: Kaufman HE, Barron BA, McDonald MB, eds. The cornea, 2nd ed. Oxford: Butterworth-Heinemann, 1998:525-50.

6 Rabinowitz Y. Keratoconus. Surv Ophthalmol 1998:42:297-319.

7 Lass JH, Lembach RG, Park SB, et al. Clinical management of keratoconus: a multi-center analysis. Ophthalmology 1990;97:433-45.

8 Crews MJ, Driebe WT, Stern GA. The clinical management of keratoconus: a 6 year retrospective study. CLAO J 1994:20:194-7.

9 Gordon MO, Schechtman KB, Davis $\amalg$, et al. Visual acuity repeatability in keratoconus: impact on sample size. Collaborative Longitudinal Evaluation of Keratoconus (CLEK) Study Group. Optom Vis Sci 1998;75:249-57.

10 McLeod SD. Beyond snellen acuity: the assessment of visual function after refractive surgery. Arch Ophthalmol 2001;119:1371-3.

11 Hess RF, Carney LG. Vision through an abnormal cornea: a pilot study of the relationship between visual loss from corneal distortion, corneal edema, keratoconus and some allied corneal pathology. Invest Ophthalmol Vis Sci 1979; 18:476-83.

12 Carney LG. Visual loss in keratoconus. Arch Ophthalmol 1982;100:1282-5.

13 Carney LG. Contact lens correction of visual loss in keratoconus. Acta Ophthalmol 1982;60:795-802.

14 Zadnik K, Mannis MJ, Johnson CA, et al. Rapid contrast sensitivity assessment in keratoconus. Am J Optom Physiol Opt 1987;64:693-7.

15 Carney LG, Jacobs RJ. Problems remaining after keratoplasty for keratoconus. Clin Exp Optom 1989;72:22-5.

16 Carney LG, Lembach RG. Management of keratoconus: comparative visua assessments. CLAO J 1991;17:52-8.

17 Carney LG, Kelly CG. Visual losses after myopic epikeratoplasty. Arch Ophthalmol 1991;109:499-502.

18 Rabin J, Wicks J. Measuring resolution in the contrast domain: the small letter contrast test. Optom Vis Sci 1996;73:398-403.

19 Applegate RA, Hilmantel G, Howland HC, et al. Corneal first surface optical aberrations and visual performance. J Refract Surg 2000;16:507-14.

20 Maeda N, Sato S, Watanabe H, et al. Prediction of letter contrast sensitivity using videokeratographic indices. Am J Ophthalmol 2000;129:759-63.

21 Rose K, Harper R, Tromans C, et al. Quality of life in myopia. Br J Ophthalmol 2000;84:1031-4

22 Brahma A, Ennis F, Harper R, et al. Visual function after penetrating keratoplasty for keratoconus: a prospective longitudinal evaluation. Br J Ophthalmol 2000;84:60-6.

23 Barr JT, Yackels T. Corneal scarring in keratoconus-measurements and influence on visual acuity. Int Contact Lens Clin 1991;22:173-5.

24 Zadnik K, Barr JT, Edrington TB, et al. Corneal scarring and vision in keratoconus: a baseline report from the Collaborative Longitudinal Evaluation of Keratoconus (CLEK) Study. Cornea 2000;19:804-12.

25 Betts AM, Mitchell GL, Zadnik K. Visual performance and comfort with the Rose K lens for keratoconus. Optom Vis Sci 2002;79:493-501.

26 Mannis MJ, Zadnik K, Johnson CA, et al. Contrast sensitivity after penetrating keratoplasty. Arch Ophthalmol 1987;105:1220-3.

27 Khong AM, Mannis MJ, Plotnik RD, et al. Computerised topographic analysis of the healing graft after penetrating keratoplasty for keratoconus. Am J Ophthalmol 1993;115:209-15.

28 Mannis MJ, Zadnik K, Johnson CA. The effect of penetrating keratoplasty on contrast sensitivity in keratoconus. Arch Ophthalmol 1984;102:1513-6.

29 Zadnik KM, MJ Johnson, CA. An analysis of contrast sensitivity in identical twins with keratoconus. Cornea 1984;3:99-103.

30 Miller D, Sanghvi S. Contrast sensitivity and glare testing in corneal disease. In: Nadler MP, Miller D, Nadler DJ, eds. Glare and contrast sensitivity for clinicians. New York: Springer Verlag, 1990:45-52.

31 Krumeich JH, Daniel J, Knulle A. Live-epikeratophakia for keratoconus. $J$ Cataract Refract Surg 1998;24:456-63.

32 Lenne RC, Smith G, Vingrys AJ. Automated visual acuity testing. Clin Exp Optom 1994;77:190-5.

33 Regan D, Giaschi DE, Fresco BB. Measurement of glare sensitivity in cataract patients using low-contrast letter charts. Ophthal Physiol Opt 1993;13:115-23.

34 Laidlaw DAH, Haddad R. Can second eye cataract surgery be justified? Eye 1993;7:680-6.

35 Pelli DG, Robson JG, Wilkins AJ. The design of a new letter chart for measuring contrast sensitivity. Clin Vis Sci 1988;2:187-99.

36 Elliott DB, Sanderson K, Conkey A. The reliability of the Pelli-Robson contrast sensitivity chart. Ophthal Physiol Opt 1990;10:21-4.

37 Elliott DB, Bullimore MA. Assessing the reliability, discriminative ability, and validity of disability glare tests. Invest Ophthalmol Vis Sci 1993:34:108-19.

38 Pesudovs K, Hazel CA, Doran RML, et al. The usefulness of Vistech and FACT contrast sensitivity charts for cataract and refractive surgery outcomes research. Br J Ophthalmol 2004;88:11-16.

39 Elliott DB, Bullimore MA, Bailey IL. Improving the reliability of the Pelli-Robson contrast sensitivity test. Clin Vis Sci 1991;6:471-5.

40 Pesudovs K, Coster DJ. Assessment of visual function in cataract patients with a mean visual acuity of 6/9. Aust NZ J Ophthalmol 1996;24:S5-9.

41 Bullimore MA, Olson MD, Maloney RK. Visual performance after photorefractive keratectomy with a 6-mm ablation zone. Am J Ophthalmol 1999;128:1-7. 
42 Wicker D, Sanislo S, Green DG. Effect of contact lens correction of sine wave contrast sensitivity in keratoconus patients after penetrating keratoplasty. Optom Vis Sci 1992;69:342-6.

43 Mannis MJ, Zadnik K, Johnson C, et al. Contrast sensitivity after epikeratoplasty. Cornea 1988;7:280-4.

44 Calver RI, Cox MJ, Elliott DB. Effect of aging on the monochromatic aberrations of the human eye. J Opt Soc Am A 1999;16:2069-78.

45 Haegerstrom-Portnoy G, Brabyn J, Schneck ME, et al. The SKILL Card: an acuity test of reduced luminance and contrast. Invest Ophthalmol Vis Sci 1997;38:207-18.
46 Foster PJ, Buhrmann R, Quigley HA, et al. The definition and classification of glaucoma in prevalence surveys. $\mathrm{Br} J$ Ophthalmol 2002;86:238-42.

47 Holland DR, Maeda N, Hannush SB, et al. Unilateral keratoconus. Incidence and quantitative topographic analysis. Ophthalmology 1997; 104:1409-13.

48 Bawazeer AM, Hodge WG, Lorimer B. Atopy and keratoconus: a multivariate analysis. Br J Ophthalmol 2000;84:834-6.

49 Colin J, Velou S. Current surgical options for keratoconus. J Cataract Refract Surg 2003;29:379-86. 